\title{
THE SUBSCRIPT OF $\boldsymbol{\aleph}_{n}$, PROJECTIVE DIMENSION, AND THE VANISHING OF $\lim _{\leftarrow}(n)$
}

\author{
BY BARBARA L. OSOFSKY
}

In this sketch, I will try to indicate how cardinality questions and manipulations became intimately connected with homological algebra. The modern form of this subject stems from the book Homological algebra, by H. Cartan and S. Eilenberg, published in 1956. What began as a study of dimension via derived functors branched off into a study of dimension via cardinality and came back to a study of derived functors via cardinality. I will give an historical sketch of this. All rings are associative with 1, all modules are unital right modules unless otherwise stated.

1. Projective dimension and Ext. Let us begin by defining the main topic of concern, projective dimension. Let $M$ be an $R$-module. Then we can express $M$ as a quotient of a free $R$-module; for example take the obvious epimorphism from $P_{0}=\bigoplus_{x \in M} R_{x}$ to $M$. One gets a short exact sequence of modules

$$
0 \rightarrow K_{1} \rightarrow P_{0} \rightarrow M \rightarrow 0 .
$$

If one defines an equivalence relation on the category of right $R$-modules by $A \sim B$ if and only if there exist free $R$-modules $P$ and $P^{\prime}$ such that $A \oplus P \approx B \oplus P^{\prime}$, then the equivalence class of $K_{1}$ depends only on the equivalence class of $M$. Now iterate this procedure to get a family of short exact sequences

$$
\begin{gathered}
0 \rightarrow K_{1} \rightarrow P_{0} \rightarrow M \rightarrow 0 \\
0 \rightarrow K_{2} \rightarrow P_{1} \rightarrow K_{1} \rightarrow 0 \\
\cdot \\
\cdot \\
\cdot \\
0 \rightarrow K_{n} \rightarrow P_{n-1} \rightarrow K_{n-1} \rightarrow 0
\end{gathered}
$$

An invited address delivered to the seventy-ninth annual meeting in Dallas, Texas on January 25, 1973; received by the editors May 27, 1973.

AMS (MOS) subject classifications (1970). Primary 16A50, 16A60, 18G10, $18 \mathrm{G} 20$.

Key words and phrases. Projective dimension, derived functors. 
or, all put together, one long exact sequence

$$
\mathscr{P}: \cdots \rightarrow P_{n} \rightarrow \cdots \rightarrow P_{1} \rightarrow P_{0} \rightarrow M \rightarrow 0
$$

called a projective resolution of $M$. (The $P_{i}$ must be projective, but not necessarily free.) The projective dimension of $M, \operatorname{pd}(M)$, is the smallest $n$ such that $K_{n} \in$ class (0) (i.e., $K_{n}$ is projective) or $\infty$ if no such $n$ exists. Alternatively, one can use the derived functors Ext of Hom. For any right $R$-module $A$, we hom each of the short exact sequences $(*)$ into $A$. Since $\operatorname{Hom}(, A)$ is left exact but not exact, we have cokernels, called Ext

$0 \rightarrow \operatorname{Hom}(M, A) \rightarrow \operatorname{Hom}\left(P_{0}, A\right) \rightarrow \operatorname{Hom}\left(K_{1}, A\right) \rightarrow \operatorname{Ext}^{1}(M, A) \rightarrow 0$

$0 \rightarrow \operatorname{Hom}\left(K_{1}, A\right) \rightarrow \operatorname{Hom}\left(P_{1}, A\right) \rightarrow \operatorname{Hom}\left(K_{2}, A\right) \rightarrow \operatorname{Ext}^{2}(M, A) \rightarrow 0$

$0 \rightarrow \operatorname{Hom}\left(K_{n}, A\right) \rightarrow \operatorname{Hom}\left(P_{n}, A\right) \rightarrow \operatorname{Hom}\left(K_{n+1}, A\right) \rightarrow \operatorname{Ext}^{n+1}(M, A) \rightarrow 0$

Now if $K_{n}$ is projective, $0 \rightarrow K_{n+1} \rightarrow P_{n} \rightarrow K_{n} \rightarrow 0$ is split exact, so $\operatorname{Ext}^{n+1}(M, A)=0$. Moreover, if $\operatorname{Ext}^{n+1}\left(M, K_{n+1}\right)=0,0 \rightarrow K_{n+1} \rightarrow P_{n}$ splits, so $K_{n}$ is projective. We thus have

$$
\operatorname{pd}(M) \leqq n \Leftrightarrow \operatorname{Ext}^{n+1}(M, A)=0
$$

for all modules $A$, where $\operatorname{Ext}^{*}(M, A)$ is the homology of the complex

$$
\begin{aligned}
\operatorname{Hom}_{R}(\mathscr{P}, A): 0 & \rightarrow \operatorname{Hom}_{R}\left(P_{0}, A\right) \rightarrow \operatorname{Hom}_{R}\left(P_{1}, A\right) \rightarrow \cdots \\
& \rightarrow \operatorname{Hom}_{R}\left(P_{n}, A\right) \rightarrow \cdots
\end{aligned}
$$

The (right) global dimension of $R$ is the supremum of the projective dimensions of all right $R$-modules. The global dimension of $R \leqq n \Leftrightarrow$ the bifunctor $\operatorname{Ext}^{n+1}(A, B)$ vanishes identically.

There is another important dimension obtained from a projective resolution of $M$, namely the weak dimension of $M$. This is the first $n$ such that $K_{n}$ is flat $\left(K_{n} \otimes_{R}\right.$ is exact), or the first $n$ such that $\operatorname{Tor}_{n+1}(M,) \equiv$ 0 where $\operatorname{Tor}_{*}(M, A)$ is the homology of the sequence

$$
\cdots \rightarrow P_{n} \otimes_{R} A \rightarrow \cdots \rightarrow P_{0} \otimes_{R} A \rightarrow 0 .
$$

The weak global dimension of $R$ is the smallest $n$ such that $\operatorname{Tor}_{n+1}(A, B) \equiv$ 0 . Whereas Ext involves modules on only one side, Tor involves both right and left modules. Since there is a "commutativity" property for 
derived functors of bifunctors, the left and right weak global dimensions of a ring are equal, but there was no reason to believe the same was true for global dimensions (indeed it is not) and Cartan-Eilenberg posed the question of equality in Chapter 1 of their book on homological algebra [8].

A word about the definition. This kind of thing is the standard fare of algebraic topology. In algebraic form it can be found in the famous

THEOREM (HILBERT SYZYGY THEOREM, 1890 [19]). Let $A$ be a graded module over the graded polynomial ring $R=F\left[x_{1} \cdots x_{n}\right]$ in $n$ indeterminants (of degree 1) over a field $F$. Then any exact sequence

$$
0 \rightarrow K_{n} \rightarrow P_{n-1} \rightarrow \cdots \rightarrow P_{0} \rightarrow A \rightarrow 0
$$

of graded $R$-modules with the $P_{i}$ free has its $n$th term $K_{n}$ free.

The word syzygy just means module of relations. Here not only projectivity, but freeness of the $n$th syzygy is asserted. Hilbert was concerned with modules of forms invariant under a group of linear transformations so the graded restriction was natural. This result lay dormant for a while, until Gröbner (1949) [16] simplified Hilbert's proof, and Koszul (1950) [32] put the result in a modern topological setting. Cartan (1952) [7] first applied homological methods to generalize the theorem. The terminology Ext, for extensions, was introduced by Eilenberg and Mac Lane in 1942 [13] in studying the universal coefficient theorem of topology. $\operatorname{Ext}(M, A)$ can be interpreted as extensions of $A$ by $M$, that is, short exact sequences $0 \rightarrow A \rightarrow E \rightarrow M \rightarrow 0$ modulo an appropriate equivalence relation.

These were just some of the historical developments prior the advent of the Cartan-Eilenberg book. Even before it appeared in print, Homological algebra exerted its influence on the new subject it was launching, tying together various homological and cohomological dimensions as in topology, groups, Lie algebras, the previously named Ext $\left(=\operatorname{Ext}^{1}\right)$ and Tor $(=$ torsion product), and setting the notation (such as $\mathrm{Ext}^{n}$ ) and ideas pervading the subject thereafter. This book marks the real beginning of the subject of homological algebra as an area of interest in its own right. It also makes the derived functors Ext (and Tor) the "natural" way of looking at homological dimension.

2. Some examples and early results. Let us look at some examples of low dimensions (with terminology adapted to the modern context).

THEOREM (WEDDERBURN-ARTIN). The following are equivalent:

(i) $\mathrm{gl} \mathrm{d}(R)=0$.

(ii) $R$ is a finite ring direct product of matrix rings over division rings.

(iii) $R$ has minimum condition on right ideals and no nilpotent ideals. 
THEOREM. Let $R$ be a commutative domain. Then $R$ has global dimension $\leqq 1$ if and only if $R$ is a Dedekind domain (the kind axiomatically studied by E. Noether in 1927 [43] distilling the essential properties of algebraic number rings).

THEOREM (HochSCHILD, 1946 [21]). Let $F$ be a field, $R$ a finitedimensional F-algebra. Then $R$ is separable (that is, for all extension fields $L \supseteq F, R \otimes_{F} L$ is semisimple with minimum condition) if and only if

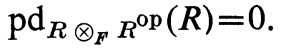

Let us look at one of the early results in projective dimension theory and how it was proved. The Nagoya Journal had a whole series of papers "On the dimensions of modules and algebras" in the 1950's. Much of the work involved Ext and Tor (and special rings such as semiprimary and hereditary rings). Number 3 in the series had a large portion of this type. However, the first sections were of a different nature, and it is they that interest us here.

Proposition (Auslander, 1955 [1]). Let $A$ be an $R$-module, I a nonempty well-ordered set and $\left\{A_{i} \mid i \in I\right\}$ a family of submodules of $A$ such that for all $i, j \in I, i \leqq j \Rightarrow A_{i} \subseteq A_{j}$. If $A=\bigcup_{i \in I} A_{i}$ and $\operatorname{pd}\left(A_{i} / \bigcup_{j<i} A_{j}\right) \leqq n$ for all $i \in I$, then $\operatorname{pd}(A) \leqq n$.

This proposition has several corollaries, the first two of which were Auslander's aim in proving the proposition.

Corollary 1 (GLOBAl Dimension theOREM). Gl d $(R)=\sup \{p d(R / I) \mid I$ a right ideal of $R$ \}.

COROLlaRY 2. Let $R$ be a left and right noetherian ring (i.e. every one sided ideal is finitely generated). Then left $\mathrm{gl} \mathrm{d}(R)=$ weak $\mathrm{gl} \mathrm{d}(R)=$ right $\mathrm{gl} \mathrm{d}(R)$.

The first corollary is derived by well ordering a set of generators for an $R$-module $A$ and setting $A_{\alpha}=\sum_{\beta<\alpha} x_{\beta} R$. The second follows from the first since finitely presented flat modules are projective, so in the noetherian case $\operatorname{pd}(R / I)=$ weak dimension $(R / I)$.

Our third corollary was not stated by Auslander, but is immediate from his proposition and the result that for any short exact sequence

$$
0 \rightarrow A \rightarrow B \rightarrow C \rightarrow 0
$$

$\operatorname{pd}(A) \leqq k$ and $\operatorname{pd}(B) \leqq k$ implies $\operatorname{pd}(C) \leqq k+1$. This is provable trivially using the long exact sequence for Ext, but also not difficult to do directly by an induction argument. 
COROLlaRY 3. Let $M$ be an R-module generated by $\aleph_{n}$ elements for $n \in \omega$. Assume there exists a family of submodules of $M,\left\{N_{\alpha} \mid \alpha \in \mathfrak{I}\right\}$ directed under $\subseteq$ such that $M=\sum_{\alpha \in \mathfrak{I}} N_{\alpha}$ and $\operatorname{pd}\left(N_{\alpha}\right) \leqq k$ for all $\alpha \in \mathfrak{I}$. Then $\operatorname{pd}(M) \leqq k+n+1$. If, in addition, $\left\{N_{\alpha} \mid \alpha \in \mathfrak{I}\right\}$ is closed under unions of countable chains (respectively chains of length $\boldsymbol{\aleph}_{p}$ ), then $\operatorname{pd}(M) \leqq k+n$ (resp. $k+n-p$ ).

That this corollary to Auslander's proposition was considered obvious was apparent from an early reference. Kaplansky, in 1958 [29], constructed an example of a ring which had right global dimension 1 and left global dimension $>1$. Then Kaplansky went on to say that a regular ring of cardinality $\aleph_{1}$ (his example was regular of cardinality $2 \boldsymbol{\aleph}_{0}$ ) has global dimension at most 2 by a theorem of M. Auslander. The reference was to Corollary 3, which Kaplansky felt so obvious he did not have to state it.

This proposition of Auslander and Corollary 3 and its variants are to this day the major way of getting upper bounds on projective dimensions, at least as far as cardinalities $>\boldsymbol{\aleph}_{0}$ are concerned. But connections between projective dimensions and cardinals $>\boldsymbol{\aleph}_{0}$ were very far from the major applications of homological dimension during the next decade. For one thing, the influence of derived functors was so strong that, when Matlis (1959) [37] came out with a short, elegant proof of the global dimension theorem using properties of Ext, it completely replaced the Auslander proof as "standard fare". Matlis pointed out that, by a standard test for injectivity, $M_{R}$ is injective if and only if $\operatorname{Ext}^{1}(R / I, M)=0$ for all right ideals $I$. Using "commutativity" of Ext,

$$
\operatorname{Ext}^{n+1}(, M)=0 \Leftrightarrow \operatorname{Ext}^{n+1}(R / I, M)=0
$$

for all right ideals $I \subseteq R$ so

$$
\operatorname{Ext}^{n+1} \equiv 0 \Leftrightarrow \operatorname{Ext}^{n+1}(R / I, \quad)=0 \text { for all } I \subseteq R \text {. }
$$

In Mac Lane's Homology [35], it is Matlis' proof that is given. Jans in the summer of 1961 gave the Auslander proof of the global dimension theorem in lectures at the University of Oklahoma [22]. When these lectures were modified into his 1964 book Rings and homology [23], Matlis' proof was given. Elegant as it was, the Matlis proof of the global dimension theorem completely bypassed Auslander's proposition and its consequences for upper bounds on dimensions.

3. A major success-regular local rings. All of this was overshadowed by the fact that homological dimension was having its biggest successes elsewhere, in particular in the study of regular local rings in commutative algebra. The study of commutative noetherian rings and finitely generated modules over them had a long and honorable history. The classical work 
of E. Noether (1921) [42] set it off on axiomatic terms, but its connections with algebraic geometry, number theory, polynomials over a field, etc. were what led to this work. In 1938 [33], Krull recognized the significance of a certain class of commutative noetherian local rings, namely those where the number of generators of the maximal ideal $M$ was equal to the height of $M$ (=Krull dimension of the ring). These later came to be referred to as regular (groan) local rings. (What an abused word "regular" has become. It was used earlier in this paper with an entirely different meaning which preceded the commutative algebra nomenclature. And its uses elsewhere in mathematics are myriad.) In algebraic geometry, regularity corresponds to nonsingularity of a point on an algebraic variety (Zariski, 1947 [54]). For the regular local rings arising in algebraic geometry, Zariski showed that $R$ is a unique factorization domain and for every prime ideal $P$ of $R, R_{P}$ is regular. Krull in 1938 had asked if these properties held for arbitrary regular local rings. I. S. Cohen (1946) [9] answered the questions affirmatively for other special cases, but it remained for homological dimension theory to settle the general questions. The landmark theorem was

Theorem (Auslander-Buchsbaum, 1957 [3], Serre, 1956 [51]). Let $R$ be a commutative noetherian local ring with maximal ideal $M$. Then the following are equivalent.

(i) $R$ is regular local of dimension $n$ ( $n=$ ht $M=$ minimum number of generators of $M)$.

(ii) $\operatorname{pd}(R / M)=n$.

(iii) $\mathrm{gl} \mathrm{d}(R)=n$.

COROllary. Let $R$ be a regular local ring, $P$ a prime ideal of $R$. Then $R_{P}$ is a regular local ring of dimension $=$ ht $P$.

Homological methods also led to the solution of the unique factorization problem of Krull which Nagata had reduced to the case of dimension 3.

Theorem (Auslander-Buchsbaum, 1959 [4]). A regular lócal ring is a unique factorization domain.

With such great successes obtained by its use, homological dimension theory was firmly entrenched in the study of finitely generated modules over commutative noetherian rings. Here the problems of infinite cardinals were completely irrelevant. Not even choice was necessary-the ascending chain condition replaced Zorn's lemma.

4. Cardinality comes in. Although the use of derived functors and the applications in the noetherian case almost completely eclipsed cardinality questions in homological dimension, there were some results in 
the countable case during this time. Berstein (1958) [6] showed that a countable direct limit of modules of projective dimension $\leqq k$ had dimension $\leqq k+1$. Jensen (1966) [25] showed that a countably related flat module had dimension $\leqq 1$. This result could also be obtained from Berstein's result and Lazard's theorem that a flat module is a direct limit of finitely generated free modules [34]. (The indexing set size can be reduced.) By manipulating to get the form of the third corollary to Auslander's proposition, Osofsky (1968) [47] extended these results to say that if $D$ is a directed set of cardinality $\boldsymbol{\aleph}_{u}$, then

$$
\operatorname{pd}\left(\lim _{\rightarrow} M_{i}\right) \leqq \sup \left\{\operatorname{pd}\left(M_{i}\right) \mid i \in D\right\}+n+1
$$

and for any $\boldsymbol{\aleph}_{n}$-related flat module $M$,

$$
\operatorname{pd}(M) \leqq n+1 \text {. }
$$

This pointed out the foresight in Kaplansky's observation on constructing rings of differing right and left global dimensions. As a corollary, any ring of cardinality $\boldsymbol{\aleph}_{n}$ has global dimension and weak global dimension differing by at most $n+1$, so left and right global dimensions can differ by at most $n+1$. To see the bound on gl d-weak gl d, resolve a cyclic. At the weak global dimension step you have an $\aleph_{n}$-generated flat kernel, with p.d. at most $n+1$. Small in 1966 [53] used Kaplansky's example to construct a ring with left and right global dimensions differing by 2 , but that is as far as you can go in Zermelo-Frankel plus Choice + Continuum Hypothesis with a ring of cardinality $2^{\aleph_{0}}$.

The first irrefutable indications that cardinality was intimately tied up with projective dimension came in 1967 in two separate papers where lower bounds as well as upper bounds on dimensions were calculated in terms of subscripts of cardinalities. For convenience we let $\boldsymbol{\aleph}_{-1}$ denote any finite cardinal and $\infty$ any cardinal $\geqq \omega$.

Theorem (Pierce, 1967 [48]). Let $R$ be a free Boolean ring on $\boldsymbol{\aleph}_{n}$ generators. Then $\mathrm{gl} \mathrm{d}(R)=n+1$.

THEOREM (OSOFSKY, 1967 [44]). Let $R$ be a valuation domain, I an ideal of $R$. Then $\operatorname{pd}(I)=n+1 \Leftrightarrow I$ is generated by $\aleph_{n}$ but no fewer elements.

This theorem holds if $R$ is any ring and $I$ any module with a linearly ordered set of free generators with a condition insuring it holds for $n=0$.

The upper bounds indicated in these theorems are consequences of Auslander's proposition (although in the second paper a different technique was used). Both papers used a specific projective resolution to get a lower bound. The resolutions were similar but not identical. As these same resolutions were used to get other lower bounds, I will postpone discussion of them until a later point. 
These results have a strong tie-in with the continuum hypothesis, but only in an artificial way. You are plugging in the cardinality. I know of no "natural" way to get a valuation domain possessing an ideal generated by $\aleph_{n}$ but no fewer elements without essentially plugging in the $\boldsymbol{\aleph}_{n}$.

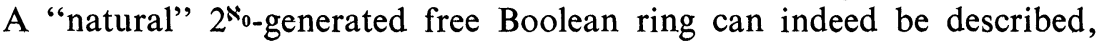
but is not at all "obvious".

In the next results, however, the continuum hypothesis is there, loud and clear, even though the phraseology is still in terms of $\boldsymbol{\aleph}_{n}$.

THEOREM (OSOFSKY, 1970 [46]). Let $\left\{R_{i} \mid i \in \mathscr{I}\right\}$ be a family of rings such that $R$ contains the ring direct product $\prod_{i \in \mathscr{I}} R_{i}$ as a subring. If $\left|2^{\mathscr{J}}\right|=\aleph_{n}$, then $R$ contains a right ideal I generated by idempotents (characteristic functions of subsets of $\mathscr{I})$ such that $\operatorname{pd}(I)=n$.

COROLlary. Let $\left\{F_{i} \mid i \in \omega\right\}$ be a family of fields. Then

$$
\operatorname{gld}\left(\prod_{i=0}^{\infty} F_{i}\right)=n+1 \Leftrightarrow 2 \aleph_{0}=\aleph_{n}
$$

THEOREM (OSOFSKY, 1968 [45]). Let $R$ be a ring of cardinality $\aleph_{n}$ and global dimension $k$ such that either

(i) $R$ is regular local and $|R|=|R / M|$,

(ii) $R$ is complete regular local, or

(iii) $R=F\left[x_{1}, \cdots, x_{k}\right], F$ a field.

Let $Q$ be the quotient field of $R$. Then $\operatorname{pd}(Q)=\min (k, n+1)$.

For any local domain $R$ and for "large" polynomial rings, Kaplansky showed $\operatorname{pd}(Q)=1 \Leftrightarrow Q_{R}$ countably generated in 1966 [31]. The proof of this theorem used his technique.

COROLlary.

$\operatorname{pd}\left(\boldsymbol{R}(x, y, z)_{\mathbf{R}[x, y, z]}\right)=2 \Leftrightarrow 2^{\boldsymbol{N}_{0}}=\boldsymbol{\aleph}_{1} \Leftrightarrow \operatorname{pd}\left(\boldsymbol{Q}((x, y, z))_{Q[[x, y, z] !}\right)=2$.

Upper bounds for the results in $\S 4$ are, as indicated earlier, from Corollary 3 to Auslander's proposition. The necessary lower bounds are obtained by looking at specific projective resolutions with appropriate induction steps. The corollaries indicate that we are talking about things that "exist in the real world", not just strange $\aleph$ 's that are pulled out of a foundational hat. The cardinalities in question are $2^{\boldsymbol{x}_{0}}$; the relevance to where it lies in the well ordering of the cardinals is purely a consequence of the results.

5. Technical sketch of the method of proof of cardinality results. This section may be lightly skimmed over or skipped on first reading by the reader not interested in technical details. More general discussion resumes 
in $\S 6, p .18$. Let me give you an idea of what is involved in the proofs of the necessary lower bounds. The proofs are by finite induction.

In the case of the first theorem (Osofsky, 1970 [46]), we look at right ideals generated by commuting idempotents. We throw on an extra condition to handle the case $n=0$ and prevent collapse of generators in the inductive step. This independence condition reduces to the condition that the idempotents together with 1 form a free Boolean ring under addition $e+f-e f$ and multiplication as in the ring. In any product indexed by $\mathscr{I}$, there is such a set of cardinality $\left|2^{\mathscr{I}}\right|$, giving the claimed results.

Specifically, one gets a family of idempotents $\left\{e_{i} \mid i \in \mathfrak{I}\right\}$ such that for all $i, j \in \mathfrak{J}, e_{i} e_{j}=e_{j} e_{i}$ and for disjoint finite subsets $\left\{e_{l} \mid 1 \leqq l \leqq m\right\}$ and $\left\{\left.e_{j}\right|_{m+1} \leqq j \leqq k\right\}$,

$$
\prod_{l=1}^{m} e_{l} \prod_{j=m+1}^{k}\left(1-e_{j}\right) \neq 0 .
$$

Assume $\mathfrak{J}$ is linearly ordered. Set $P_{-1}=\sum_{j \in \mathfrak{I}} e_{j} R$. Now introduce symbols $\left\langle j_{0}, \cdots, j_{n}\right\rangle$ to represent a basis for the free $R$-module indexed by $\mathfrak{I}^{n+1}$. For $n \geqq 0$, set

$$
P_{n}=\bigoplus_{j_{0}<j_{1}<\cdots<j_{n}}\left\langle j_{0}, \cdots, j_{n}\right\rangle \prod_{l=0}^{n} e_{j_{l}} R
$$

and define $d_{n}: P_{n} \rightarrow P_{n-1}$ as the map induced by $d_{0}\left\langle j_{0}\right\rangle=e_{j_{0}}$, and for $n>0$

Then

$$
d_{n}\left\langle j_{0}, \cdots, j_{n}\right\rangle=\sum_{l=0}^{n}(-1)^{l}\left\langle j_{0}, \cdots, j_{l-1}, j_{l+1}, \cdots, j_{n}\right\rangle \prod_{l=0}^{n} e_{j_{l}} .
$$

$$
\mathscr{P}: \cdots \longrightarrow P_{n} \stackrel{d_{n}}{\longrightarrow} P_{n-1} \longrightarrow \cdots \longrightarrow P_{0} \stackrel{d_{0}}{\longrightarrow} P_{-1} \longrightarrow 0
$$

is a projective resolution of $P_{-1}$. The symbol $\left\langle j_{0}, \cdots, j_{n}\right\rangle$ is an indexing notation indicating the sum is taken over the appropriate subset of $\mathfrak{J}^{n+1} .^{1}$

The projective resolution for the quotient field theorem (Osofsky, 1968 [45]), the valuation domain theorem (Osofsky, 1967 [44]), and some later applications is similar. Let $M$ be a module possessing a set of generators $\left\{x_{i} \mid i \in \mathfrak{J}\right\}$ such that

(i) $\mathfrak{J}$ is a directed set (under a relation $<$ ),

(ii) For all $r \in R$ and for all $i \in \mathfrak{J}, x_{i} r=0 \Leftrightarrow r=0$ (freeness),

(iii) $i<j \Rightarrow x_{i} R \subseteq x_{j} R$.

${ }^{1}$ This same resolution for a set of commuting idempotents satisfying a somewhat different condition has been used by Osofsky, Proc. Amer. Math. Soc. 41 (1973), 24-30, to modify Hochschild's 1946 result to the Theorem: Let $K$ be a separably generated $\boldsymbol{\aleph}_{k}$-generated field extension of a field $F$. Then $\mathrm{pd}_{K \otimes_{F} K}(K)=1+k+\operatorname{tr} \operatorname{deg}(K)$. Rosenberg and Zelinsky [50] and MacRae [36] set the stage for this result. 
Set $P_{-1}=M$. Define: For $n \geqq 0$,

$$
\begin{aligned}
P_{n}= & j_{j_{0}<\cdots<j_{n}}\left\langle j_{0}, \cdots, j_{n}\right\rangle R, \\
d_{0}\left\langle j_{0}\right\rangle= & x_{j_{0}}, \\
d_{n}\left\langle j_{0}, \cdots, j_{n}\right\rangle= & \sum_{l=0}^{n-1}(-1)^{l}\left\langle j_{0}, \cdots, j_{l-1}, j_{l+1}, \cdots, j_{n}\right\rangle \\
& +(-1)^{n}\left\langle j_{0}, \cdots, j_{n-1}\right\rangle x_{j_{n}}^{-1}\left(x_{j_{n-1}}\right),
\end{aligned}
$$

where $x_{j_{n}}^{-1}\left(x_{j_{n-1}}\right)$ is that unique element in $R$ such that $x_{j_{n}} x_{j_{n}}^{-1}\left(x_{j_{n-1}}\right)=x_{j_{n-1}}$. Then

$$
\cdots \rightarrow P_{n} \rightarrow \cdots \rightarrow P_{0} \rightarrow M \rightarrow 0
$$

is a projective resolution of $M$.

In the valuation ring example, $M$ is the ideal whose dimension is to be calculated; in the calculation of the dimension of a quotient field, $M$ is a specific kind of submodule of $Q$ and the $x_{i}$ are reciprocals of a nonzero multiplicatively closed set $\mathfrak{J}$.

In both projective resolutions, for the case of commuting idempotents and for the case of directed torsion-free modules over a domain, assuming $d_{k} P_{k}$ is projective, one uses a snaking argument of Kaplansky (1958) [30] to get a direct summand of $d_{k} P_{k}$ of the form

$$
d_{k}\left(\underset{j_{0}<\cdots<j_{k}}{\bigoplus_{0}}\left\langle j_{0}, \cdots, j_{k}\right\rangle\left(\prod_{l=0}^{k} e_{j_{l}}\right) R\right)
$$

where the $j_{l}$ are taken over some subset $\mathfrak{L}$ of $\mathfrak{J}$. Write this as $d_{k} P_{l c}(\mathfrak{L})$. Transfinite induction will give such an $\mathfrak{L}$ for any cardinal $<|\mathfrak{I}|$. Hopefully one can then use induction on $n$, where $|\mathfrak{I}|=\boldsymbol{\aleph}_{n}$. We need two things, a basis and an induction step.

For the basis in the idempotent generated ideal case, we must have $|\mathfrak{I}| \neq \aleph_{0}$ implies $P_{-1}$ is not projective. That is one place we use the hypothesis $\prod_{l=1}^{m} e_{l} \prod_{l=m+1}^{n}\left(1-e_{l}\right) \neq 0$ if the $e_{l}$ 's in the two products are distinct. The valuation domain and polynomial ring basis results are straightforward; in the case of a regular local ring the argument is messier (Kaplansky, 1966 [31]).

The easiest induction step occurs in the case when the indexing set is linearly ordered such that no ordinal of cardinality $\leqq \boldsymbol{\aleph}_{k}$ is cofinal in it. For then one can take $\tau$ an upper bound for $\mathfrak{L}$, where $|\mathfrak{L}|=\aleph_{k}$ and $d_{k} P_{k}(\mathfrak{L})$ is a direct summand of ${ }_{x} d_{k} P$. This gives that $d_{k} P_{k}(\mathfrak{L})$ is actually a direct summand of $P_{k-1}$ so $P_{-1}(\mathfrak{L})$ has dimension $\geqq k-1$. The argument works for any module with linearly ordered free generating set $\left\{x_{i} \mid i \in \mathfrak{J}\right\}$ provided $x_{i} r=x_{j}$ implies $r$ is not a zero divisor (to insure the basis for $n=0$ ). Jategaonkar (1969) [24] used this fact to show that he could 
construct a left principal ideal domain of cardinality $\aleph_{n}$ possessing a right ideal of dimension $n+1$ for any $n$. Such a ring must have left and right global dimensions differing by $n+1$, thus completely settling the old conjecture in Cartan-Eilenberg.

In the case of the quotient field theorem, the induction step is much more difficult, as the global dimension of the ring can get in the way. Kaplansky's 1966 argument, used in the case $n=0$, is again employed to reduce not only the cardinality but also the global dimension of the ring. The modules $M$ looked at have a condition to insure that the number of generators does not decrease when one goes to a lower dimensional quotient ring. One might note that these arguments make no reference at all to Ext.

An outstanding paper by Gruson and Raynaud (1971) [18] chooses, as an application of a discussion of descent of flatness and projectivity, to extend the quotient field result to any complete noetherian commutative domain. Here $k$ is the Krull dimension of the ring. There is no necessity to use derived functors in this result.

6. When derived functors vanish. Just because thinking of projective dimension in terms of the vanishing of the derived functor Ext was a factor in obscuring the intimate relationship between the projective dimension and cardinality of some modules, there is no reason why that latter relationship cannot be used to shed light on the vanishing of derived functors. With spectral sequences giving information on composite functors when appropriate derived functors vanish, one family of derived functors receiving attention were those associated with $\lim _{D}$ and $\lim _{D}$, where $D$ is a partially ordered set.

Let us recall the definition. Let $D$ be a partially ordered set, and let $\left\{M_{\alpha}, \Pi_{\alpha}^{\beta} \mid \alpha, \beta \in D, \alpha \leqq \beta\right\}$ be a system of $R$-modules indexed by $D$ and $R$-homomorphisms indexed by the relation $\leqq$ such that $\Pi_{\alpha}^{\beta}: M_{\alpha} \rightarrow M_{\beta}$ and $\Pi_{\beta}^{\prime} \Pi_{\alpha}^{\beta}=\Pi_{\alpha}^{\gamma}$ for $\alpha<\beta<\gamma \cdot \lim _{D} M_{\alpha}$ is an $R$-module together with a family of morphisms $\Pi_{\alpha}: M_{\alpha} \rightarrow \lim _{D} M_{\alpha}$ such that for all $\alpha<\beta$

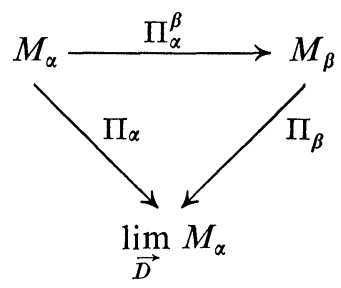

commutes and if, for a family of morphisms $v_{\alpha}: M_{\alpha} \rightarrow N, v_{\alpha}=v_{\beta} \Pi_{\alpha}^{\beta}$, then there is a unique map $g: \lim _{\rightarrow} M_{\alpha} \rightarrow N$ such that we get a 
commutative diagram

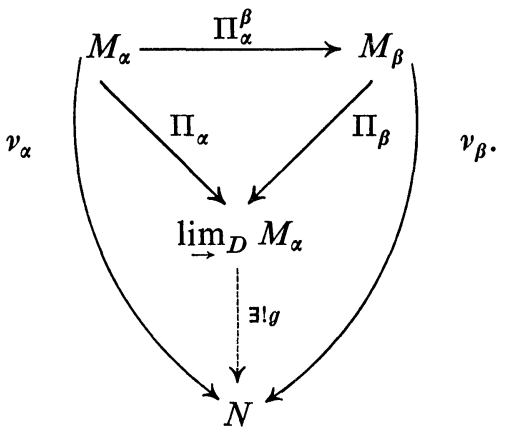

To define $\lim _{D}$, simply reverse every arrow in the above definition (so you start with an inverse system $\Pi_{\alpha}^{\beta}: M_{\beta} \rightarrow M_{\alpha}$ for $\alpha<\beta$ ).

$\lim _{D}$ and $\lim _{D}$ are functors, for let $f$ be a map from the system $\left\{M_{\alpha}\right\}$ to the system $\left\{N_{\alpha}\right\}$, that is, we have the commutative diagram

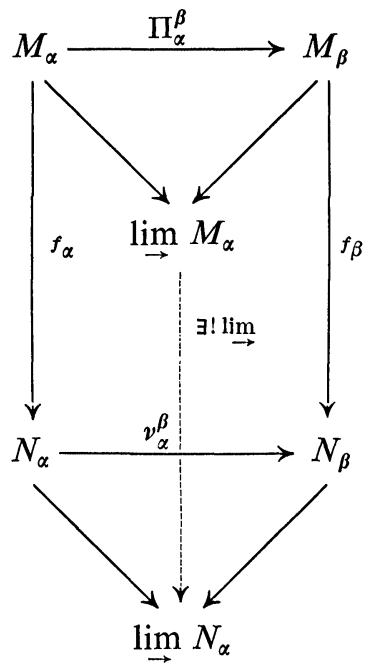

and the parallel diagram for $\lim _{D}$ with arrows reversed. These functors arise in studying the composite functors

$$
\operatorname{Hom}\left(\lim _{\rightarrow} M_{\alpha}, A\right) \approx \lim _{D} \operatorname{Hom}\left(M_{\alpha}, A\right)
$$

and $\left(\lim _{\leftarrow} M_{\alpha}\right) \otimes A$, and have topological interpretations as well. One is particularly interested in whether they vanish from some point on so one can use a convergent spectral sequence to compute derived functors. We have actually already computed the derived functors $\lim _{(k)}$ of $\lim _{D}$ for $D$ a directed set. (They are all zero for $k \geqq 1$.) The projective resolution 
constructed to compute $\operatorname{pd}(M)$ where $M$ has a partially ordered set $\left\{x_{\alpha} \mid \alpha \in \mathfrak{J}\right\}$ of free generators is, by a result of Roos (1961) [49] and Nöbeling (1962) [41], precisely the complex whose homology gives $\lim _{(k)}\left\{x_{\alpha} R \mid x_{\beta} R \subseteq x_{\alpha} R \forall \beta<\alpha\right\}$. The statement that it is exact is the statement that $\lim _{(k)}=0$ for all $k \geqq 1$ when $\mathfrak{J}$ is directed, or that the category of modules over a ring is $A B 5 . \lim _{D} M_{\alpha}$ for a partially ordered set $D$ has a somewhat similar increasing resolution whose cohomology gives $\lim _{D}^{(k)}\left\{M_{\alpha}\right\}$. Nöbeling used these sequences to calculate a bound on when $\overleftarrow{\lim }_{(k)}$ and $\lim ^{(k)}$ vanish for very special partially ordered sets. Roos $(1961) \overrightarrow{[49]}$ obtained results on the vanishing of $\lim _{D}^{(k)}$ for $k \geqq 2$ when $D$ is countable and directed, and stated that there is a natural isomorphism from the derived functors $\lim _{D}^{(k)}$ over a directed set $D$ to the derived functors $\lim _{C}^{(k)}$ over a cofinal subset $C$. A written proof (modified to cover cofinal maps which were not embeddings) appeared in C. U. Jensen's 1972 Springer Lecture Notes [28]. An even more general version with directed sets replaced by closely related categories and cofinal maps replaced by cofinal functors appeared in a paper by Mitchell (1973) [39]. Mitchell's argument is basically taken from Cartan-Eilenberg. Other results on the vanishing of $\lim _{D}^{(k)}$ were obtained under assumptions on $R$ or the modules in the inverse system or smallness of $k$ or of $|D|$, but the next really general step was

TheOREM (Goblot, 1970 [15]). Let $D$ be a directed set of cardinality $\aleph_{n}$. Then $\lim _{D}^{(n+l)}=0$ for all $l \geqq 2$.

What does all this have to do with projective dimension (besides the coincidence that a useful projective resolution is exact because $\lim _{D}$ is exact). Mitchell (1972) [38] obtained a different proof of Goblot's theorem, and at the same time showed that the bound was very precise. Indeed, he showed that if $D$ is a totally ordered set of cofinality $\boldsymbol{\aleph}_{n}$, then $\lim _{D}^{(n+1)} \neq 0$. His 1973 paper [39] with the generalization of Roos' cofinality result removes the totally ordered restriction since if the cofinality of $D$ is $\boldsymbol{\aleph}_{n}$ one can find a cofinal functor $F: D \rightarrow \Pi$ where $\Pi$ is the first ordinal of cardinality $\boldsymbol{\aleph}_{n}$. Then $F$ induces an isomorphism from $\lim _{\Pi}^{(k)}$ to $\lim _{D}^{(k)}$ so since $\lim _{\Pi}^{(n+1)}$ does not vanish, neither does $\lim _{D}^{(n+1)}$. The case $n \geqq \infty$ requires a little additional argument, which uses the same idea as the totally ordered case. We thus get the

TheOREM (MitCheLL, 1972 [38] and 1973 [39]). Let D be a directed set possessing a cofinal subset of cardinality $\boldsymbol{\aleph}_{n}$ but no cofinal subset of smaller cardinality. Then $\lim _{D}^{(n+1)} \neq 0$, but $\lim _{D}^{(n+l)}=0$ for all $l \geqq 2$.

Note that the ring $R$ is irrelevant in this result. We have sketched how to reduce the proof to the totally ordered case, but how does the proof 
in that case go? The answer is that it is identical with the calculation of the dimension of an ideal in a valuation domain, but in a more general context. After a collection of categorical definitions have been made, a student is often faced with an example-a ring is a small additive category with one object; a module is an additive functor from the ring to $\mathscr{A} b$, and $\boldsymbol{R}$-homomorphisms are natural transformations. These definitions carry over to any small additive category $\mathscr{C}$. Smallness is necessary for foundational reasons-if the objects of $\mathscr{C}$ form a proper class rather than a set, modules become proper classes and so cannot belong to any set. Indexing becomes impossible and there simply is no good way to talk about modules. Pictorially, what do we have? We have a category $\mathscr{C}$ which is a collection of objects and maps, say

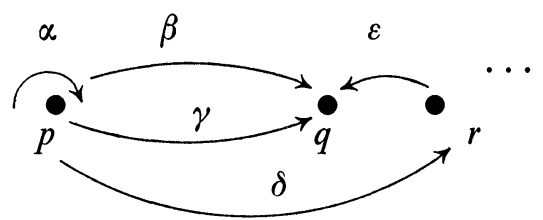

To each object we assign an abelian group, and to each map we assign a group homomorphism in an associative manner.

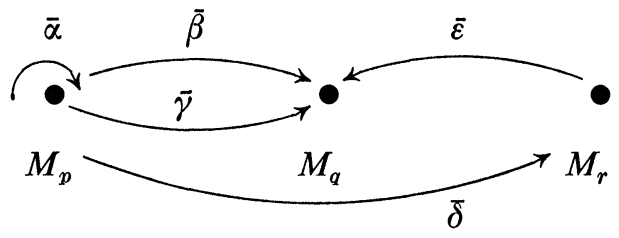

The assignment looks exactly like module multiplication by a ring element, except that you can only compose if domains and codomains agree. An element of a $\mathscr{C}$-module is an element of some $M_{p}$ (tagged by $p$ ). Let $M$ be a $\mathscr{C}$-module with a linearly ordered set of free generators $\left\{x_{i} \mid i \in \mathfrak{J}\right\}$ such that $x_{i} r=x_{j}$ implies $r$ is not a zero divisor. The ring theoretic proof that $\operatorname{pd}(M)=n+1$ if and only if the cofinality of $\mathfrak{I}$ is $\aleph_{n}$ goes through almost word for word. You have to change your thought processes slightly, but the changes in the words written down are miniscule.

How does this apply to the derived functors of $\lim$ ? Let $\Pi$ be a partially ordered set, $\Pi^{\text {op }}$ the opposite ordering of $\Pi$ interpreted as a category with a single morphism from $i$ to $j$ for all $j \leqq i$ in $\Pi$. We construct a new small category, the "category ringoid" $R \Pi^{\mathrm{op}}$ with coefficients in $R$ such that the objects in $R \Pi^{\text {op }}$ are the same as the objects in $\Pi$, and the morphism group $R \Pi^{\circ \mathrm{p}}(p, q)$ is the free $R$-module with basis the set of morphisms from $p$ to $q$ in $\Pi^{\text {op }}$. (In the partially ordered set case there is at most one such morphism, but one can do the same construction for an arbitrary 
small category, rather than just $\Pi^{\mathrm{op}}$, so we will phrase things in a more general manner.) Morphisms in $R \Pi^{\text {op }}$ are composed by: for all $\alpha: p \rightarrow q$, $\beta: q \rightarrow u \in \Pi^{\circ p}$, and for all $r, s \in R, \beta s \circ \alpha r=(\beta \circ \alpha) s r$ (plus the distributive law in a more general category where there may be more than one morphism between objects). We restrict module functors to take values in the category of $R$-modules and homomorphisms (rather than just $\mathscr{A} b$ ).

One particular $R \Pi^{\mathrm{op}}$-module is of significance. Let $\Delta R$ (technically $\Delta_{\Pi^{\text {op }}} R$ denote the functor such that

$$
\begin{array}{ll}
\Delta R(p)=R & \text { for all objects } p \text { of } \Pi, \\
\Delta R(\alpha) \text { takes } 1 \text { to } 1 & \text { for all } \alpha: p \rightarrow q \text { in } \Pi^{\text {op }} .
\end{array}
$$

Pictorially we have

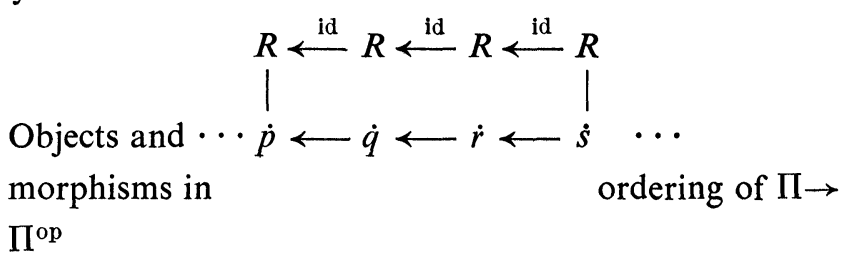

Let $M$ be any $R \Pi^{\mathrm{op}}$-module (functor from $R \Pi^{\mathrm{op}}$ to $\mathscr{M}_{R}=$ right $R$ modules). We get an inverse system of $R$-modules indexed by $\Pi$

$$
\begin{gathered}
M_{p} \stackrel{M_{p}^{q}}{\longleftarrow} M_{q} \stackrel{M_{q}^{r}}{\longleftarrow} M_{r} \stackrel{M_{r}^{s}}{\longleftarrow} M_{s} \\
\dot{p}<\dot{q}<\dot{r}<\dot{s}
\end{gathered}
$$

and we can find its $\operatorname{limit} \lim _{\Pi} M$ in the category of right $R$-modules. That is, $\lim _{\Pi}$ is a functor from $R \Pi^{\mathrm{op}}$ modules to $R$-modules. What is that functor? Let $f \in \operatorname{Hom}_{R \Pi^{\circ p}}(\Delta R, M)$, that is, $f$ is a natural transformation giving commutative diagrams of $R$-modules

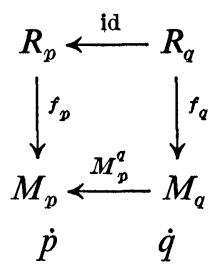

By definition of $\lim _{\Pi}$, there exists a unique map $\phi(f): R \rightarrow \lim _{\leftarrow} M$ such 
that

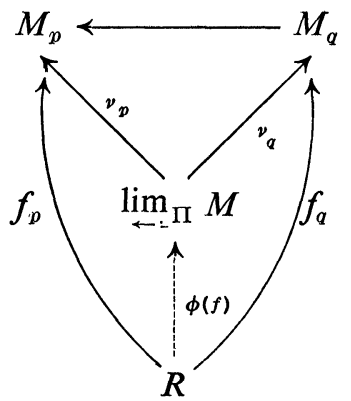

commutes. Moreover, any $R$-map from $R$ to $\lim _{\Pi} M$ gives rise to a natural transformation from $\Delta R$ to $M$ obtained by composition with the $v_{p}$. It is easy to check that $\phi$ is a natural equivalence between the functors $\operatorname{Hom}_{R \Pi^{\mathrm{op}}}(\Delta R, M)$ and $\operatorname{Hom}_{R}\left(R, \lim _{\Pi} M\right)$ which is naturally equivalent to the functor $\lim _{\Pi} M$. Since naturally equivalent functors have naturally equivalent derived functors, $\operatorname{Ext}^{n}(\Delta R, \quad)$ is naturally equivalent to $\lim _{\Pi}^{(n)}$. But $\operatorname{Ext}^{n+1}(\Delta R$,$) is identically zero if and only if \operatorname{pd}(\Delta R) \leqq n$. For each $p \in \Pi$, let $x_{p}$ be the element of $\Delta R$ consisting of 1 at object $p$ and zeros elsewhere. If $p<q$, then there is a morphism $u$ in $R \Pi^{\mathrm{op}}$ such that $x_{p}=x_{q} u$, namely $u=($ map from $q$ to $p) \cdot 1$. It is clear that $u$ is not a zero divisor, that is, if $v u$ or $u v$ is defined, it is nonzero if and only if $v$ is nonzero. Moreover, any element of $\Delta R$ (=element in some $\Delta R(p))$ is of the form $x_{p} \cdot r$ for some $r \in R \Pi^{\mathrm{op}}$, and $x_{p} \cdot r=0$ implies $r=0$. Thus the $\left\{x_{p} \mid p \in \Pi\right\}$ form a set of free generators for the module $\Delta R$. An upper bound on the projective dimension follows from Corollary 3 to Auslander's proposition and if $\Pi$ is totally ordered, $\operatorname{pd}(\Delta R)=n+1$ if and only if $\boldsymbol{\aleph}_{n}$ is the smallest cardinal cofinal in $\Pi$ if and only if $\operatorname{Ext}^{n+1}(\Delta R$,$) is the largest non-$ vanishing $\operatorname{Ext}^{(k)}(\Delta R$,$) if and only if \lim _{\Pi}^{(n+1)}$ is the largest nonvanishing derived functor of $\lim _{\Pi}$. The problem with the calculation for an arbitrary partially ordered set is the same as the problem of calculation of the projective dimension of an arbitrary module - there is no general induction step that always works as the quotient field results show (global dimension, for example, can get in the way). But there is enough information to reduce from the case of infinite $n$ to finite $n$. If the cofinality of (arbitrary directed) $\Pi \geqq \aleph_{\omega}$ and $\operatorname{pd}(\Delta R)=k<\infty$, then one can find a submodule generated by $\left\{x_{p} \mid p \in \mathfrak{L}\right\}$ where $|\mathfrak{L}|=\aleph_{k}$ and $d_{k} P_{k}(\mathfrak{L})$ is a direct summand of $d_{k} P_{k}(\Pi)$. This says $\operatorname{pd}\left(P_{-1}(\mathfrak{L})\right) \leqq k$, which contradicts the result (for finite subscripts) that $\operatorname{pd}\left(P_{-1}(\mathfrak{L})\right)=k+1$. The proof of the arbitrary case from the linearly ordered one was sketched previously.

Thus to some extent the things studied were changed, and the changed approach shed light on the original topics of interest. The Cartan-Eilenberg 
approach to projective dimension via Ext was put aside in favor of using projective resolutions directly. One of the consequences was some new information about Ext and about a family of derived functors which seemed different from Ext at first glance, but could be interpreted as an appropriate Ext. In the process one obtained the solution of several problems connecting cardinality and dimension that had previously been solved only in low cardinality cases, plus some weird homological equivalences of the continuum hypothesis.

\section{REFERENCES}

1. M. Auslander, On the dimension of modules and algebras. III. Global dimension, Nagoya Math. J. 9 (1955), 67-77. MR 17, 579.

2. M. Auslander and D. Buchsbaum, Homological dimension in Noetherian rings, Proc. Nat. Acad. Sci. U.S.A. 42 (1956), 36-38. MR 17, 705.

3. - Homological dimension in local rings, Trans. Amer. Math. Soc. 85 (1957), 390-405. MR 19, 249.

4. - Unique factorization in regular local rings, Proc. Nat. Acad. Sci. U.S.A. 45 (1959), 733-734. MR 21 \#2669.

5. R. Baer, Abelian groups which are direct summands of every containing abelian group, Proc. Amer. Math. Soc. 46 (1940), 800-806. MR 2, 126.

6. I. Bernstein, On the dimension of modules and algebras. IX. Direct limits, Nagoya Math. J. 13 (1958), 83-84. MR 20 \#7048.

7. H. Cartan, Extension du théorème des "chaînes de syzgyies", Univ. Roma Ist. Naz. Alta Mat. Rend. Mat. e Appl. (5) 11 (1952), 156-166. MR 15, 597.

8. H. Cartan and S. Eilenberg, Homological algebra, Princeton Univ. Press, Princeton, N.J., 1956. MR 17, 1040.

9. I. S. Cohen, On the structure and ideal theory of complete local rings, Trans. Amer. Math. Soc. 59 (1946), 54-106. MR 7, 509.

10. S. Eilenberg, Algebras of cohomologically finite dimension, Comment. Math. Helv. 28 (1954), 310-319. MR 16, 442.

11. - Homological dimension and syzygies, Ann. of Math. (2) 64 (1956), 328-336. MR 18, 558.

12. S. Eilenberg, M. Ikeda and T. Nakayama, On the dimensions of modules and algebras. I, Nagoya Math. J. 8 (1955), 49-57. MR 16, 993.

13. S. Eilenberg and S. Mac Lane, Group extensions and homology, Ann. of Math. (2) 43 (1942), 757-831. MR 4, 88.

14. S. Eilenberg, H. Nagao and T. Nakayama, On the dimension of modules and algebras. IV. Dimension of residue rings of hereditary rings, Nagoya Math. J. 10 (1956), 87-95. MR 18, 9.

15. R. Goblot, Sur les dérivés de certaines limites projectives, applications aux modules, Bull. Sci. Math. (2) 94 (1970), 251-255. MR 43 \#320.

16. W. Gröbner, Über die Syzgyien-Theorie der Polynomideale, Monatsh. Math. 53 (1949), 1-16. MR 11, 489.

17. A. Grothendieck, Sur quelques points d'algebre homologique, Tôhoku Math. J. (2) 9 (1957), 119-221. MR 21 \#1328.

18. L. Gruson and M. Raynaud, Critéres de platitude et de projectivité, Invent. Math. 13 (1971), 1-89. 
19. D. Hilbert, Über die Thèorie der Algebraischen Formen, Math. Ann. 36 (1890), 473-534.

20. G. Hochschild, On the cohomology groups of an associative algebra, Ann. of Math. (2) 46 (1945), 58-67. MR 6, 114.

21. - On the cohomology theory for associative algebras, Ann. of Math. (2) 47 (1946), 568-579. MR 8, 64.

22. J. P. Jans, Homological algebra and ring theory, Lecture Notes, University of Oklahoma, Stillwater, Oklahoma, 1961.

23. — Rings and homology, Holt, Rinehart and Winston, New York, 1964. MR 29 \#1243.

24. A. Jategaonkar, A counter-example in ring theory and homological algebra,

J. Algebra 12 (1969), 418-440. MR 39 \#1485.

25. C. U. Jensen, On homological dimensions of rings with countably generated ideals, Math. Scand. 18 (1966), 97-105. MR 34 \#7611.

26. - Homological dimensions of $\aleph_{0}$-coherent rings, Math. Scand. 20 (1967), 55-60. MR 35 \#2921.

27. —_ On the vanishing of $\lim ^{(i)}$, J. Algebra 15 (1970), 151-166. MR 41 \#5460.

28. - Les foncteurs dérivés de lim et leurs applications en théorie des modules, Lecture Notes in Math., no. 254, Springer-Verlag, Berlin, 1972.

29. I. Kaplansky, On the dimension of modules and algebras. X. A right hereditary ring which is not left hereditary, Nagoya Math. J. 13 (1958), 85-88. MR 20 \#7049.

30. — Projective modules, Ann. of Math. (2) 68 (1958), 372-377. MR 20 \#6453.

31. - The homological dimension of a quotient field, Nagoya Math. J. 27 (1966), 139-142. MR 33 \#2664.

32. J. L. Koszul, Sur un type d'algèbres différentielles en rapport avec la transgression. Colloque de topologie (espaces fibrés), Bruxelles, 1950; Georges Thone, Liège; Masson, Paris, 1951, pp. 73-81. MR 13, 109.

33. W. Krull, Dimensions theorie in Stellenringen, J. Reine Angew Math 179 (1938), 204-226.

34. D. Lazard, Sur les modules plats, C.R. Acad. Sci. Paris 258 (1964), 6313-6316. MR 29 \#5883.

35. S. Mac Lane, Homology, Die Grundlehren der math. Wissenschaften, Band 114, Academic Press, New York; Springer-Verlag, Berlin, 1963. MR 28 \#122.

36. R. MacRae, On cardinality, cohomology and a conjecture of Rosenberg and Zelinsky, Trans. Amer. Math. Soc. 118 (1965), 243-246. MR 31 \#2297.

37. E. Matlis, Applications of duality, Proc. Amer. Math. Soc. 10 (1959), 659-662. MR 21 \#5667

38. B. Mitchell, Rings with several objects, Advances in Math. 8 (1972), 1-161. MR 45 \#3524.

39. — The cohomological dimension of a directed set, Canad. J. Math. 25 (1973), 233-238.

40. D. Northcott, An introduction to homological algebra, Cambridge Univ. Press, New York, 1960. MR 22 \#9523.

41. G. Nöbeling, Über die derivierten des inversen und des direkten limes einer Modulfamilie, Topology 1 (1962), 47-61. MR 25 \#2109.

42. E. Noether, Idealtheorie in Ringbereichen, Math. Ann. 83 (1921), 24-66.

43. - Abstrakter Aufbou der Idealtheorie in algebraischen Zahl-und Funktionenkörpern, Math. Ann. 96 (1927), 26-61.

44. B. Osofsky, Global dimension of valuation rings, Trans. Amer. Math. Soc. 127 (1967), 136-149. MR 34 \#5899. 
45. - Homological dimension and the continuum hypothesis, Trans. Amer. Math. Soc. 132 (1968), 217-230. MR 37 \#205.

46. — Homological dimension and cardinality, Trans. Amer. Math. Soc. 151 (1970), 641-649. MR 42 \#321.

47. - Upper bounds on homological dimensions, Nagoya Math. J. 32 (1968), 315-322. MR 38 \#1128.

48. R. S. Pierce, The global dimension of Boolean rings, J. Algebra 7 (1967), 91-99. MR 37 \#5269.

49. J. E. Roos, Sur les foncteurs dérivés de lim. Applications, C.R. Acad. Sci. Paris 252 (1961), 3702-3704. MR 24 \#A1938.

50. A. Rosenberg and D. Zelinsky, Cohomology of infinite algebras, Trans. Amer. Math. Soc. 82 (1956), 85-98. MR 17, 1181.

51. J. P. Serre, Sur la dimension homologique des anneaux et des modules noethérians, Proc. Internat. Sympos, on Algebraic Number Theory, Tokyo \& Nikko, 1955; Science Council of Japan, Tokyo, 1956, pp. 175-189. MR 19, 119.

52. L. W. Small, An example in Noetherian rings, Proc. Nat. Acad. Sci. U.S.A. 54 (1965), 1035-1036. MR 32 \#5691.

53. - , Hereditary rings, Proc. Nat. Acad. Sci. U.S.A. 55 (1966), 25-27. MR 32 4178.

54. O. Zariski, The concept of a simple point of an abstract algebraic variety, Trans. Amer. Math. Soc. 62 (1947), 1-52. MR 9, 99.

Department of Mathematics, Rutgers University, New Brunswick, New JERSEY 08903 